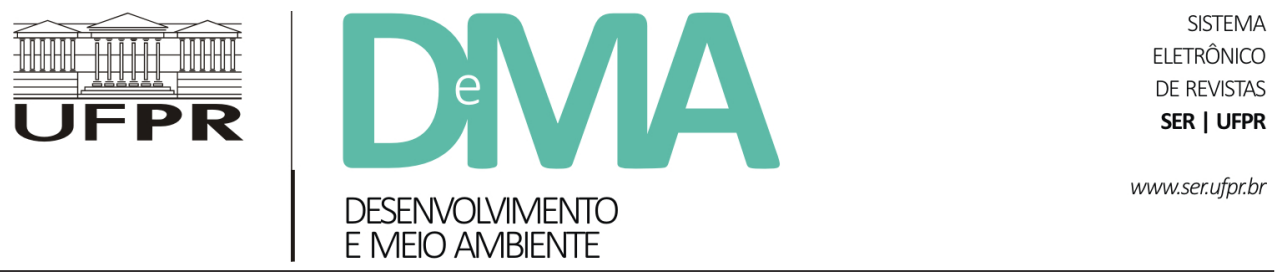

\title{
Los bancos de semillas: entre la preservación y la apropiación de recursos naturales. El acceso a los recursos fitogenéticos en la Argentina
}

\section{Bancos de sementes: entre a preservação e a apropriação dos recursos naturais. $O$ acesso aos recursos fito-genéticos na Argentina}

\section{Seed Banks: Between Natural Resources Preservation and Appropriation. Phyto-genetic Access in Argentina}

\author{
Pablo Ariel PELLEGRINI ${ }^{1,2 *}$, Galo Ezequiel BALATTI ${ }^{3}$ \\ ${ }^{1}$ Consejo Nacional de Investigaciones Científicas y Técnicas (CONICET), Ministerio de Ciencia, Tecnología e Innovación Productiva, Argentina. \\ ${ }^{2}$ Instituto de Estudios sobre la Ciencia y la Tecnología, Universidad Nacional de Quilmes, Argentina. \\ ${ }^{3}$ Universidad de Buenos Aires, Argentina. \\ *E-mail de contacto: ppellegrini@unq.edu.ar
}

Artículo recibido el 18 de mayo, 2016, versión final aceptada el 10 de mayo, 2017.

RESUMEN: En las últimas décadas fueron cobrando gran relevancia para la agricultura y la alimentación, unas instituciones poco consideradas desde las ciencias sociales: los bancos de semillas. Se trata de instituciones clave en relación a la conservación y uso de recursos naturales. En este trabajo las analizaremos a partir de un abordaje cualitativo, que combina entrevistas en profundidad a referentes de bancos de semillas en la Argentina -país donde la agricultura resulta un sector fundamental de la economía-, con el análisis de normas y documentos relacionados. Presentaremos cómo se desarrollaron las principales normas y acuerdos internacionales sobre el tema, cómo impactaron en la Argentina y qué normas propias se han implementado respecto a la conservación de recursos fitogenéticos en el país, con el fin de analizar cómo se fue construyendo el marco normativo e institucional que regula el acceso a los recursos fitogenéticos en la Argentina.

Palabras clave: biodiversidad; legislación ambiental; soberanía ambiental; FAO; ONU.

RESUMO: Nas últimas décadas, os bancos de sementes ganharam uma importância crescente no domínio da agricultura e da alimentação. No entanto, apesar de serem instituições-chave relacionadas à utilização e à conservação de recursos naturais, não foram suficientemente analisadas pelas ciências sociais. Neste trabalho, analisamos os bancos de sementes através de uma abordagem qualitativa, que combina tanto entrevistas em profundidade 
com atores referentes dos bancos de sementes na Argentina -um país onde a agricultura é um setor fundamental para a economia- coma análise de normas e documentos relacionados. Apresentaremos como foram desenvolvidas as principais normas e acordos internacionais sobre o tema, como elas têm influenciado na Argentina, e quais normas próprias têm sido implementadas sobre a conservação de recursos fitogenéticos no país, com o intuito de analisar como foi construido o quadro normativo e institucional que regula o acesso aos recurso fitogenéticos na Argentina.

Palavras-chave: biodiversidade; legislação ambiental; soberania ambiental; FAO; ONU.

ABSTRACT: Over the last decades seed banks have become highly relevant in the field of agriculture and alimentation.

Although these institutions were under-considered by social sciences, seed banks are key institutions related to the use and conservation of natural resources. In this paper they are analyzed from a qualitative approach, that combines both deeply interviews with seed banks referents in Argentina -a country with an economy fundamentally based on agriculture-, and documentation sources, among others. We will investigate how the main legal norms and international agreements were developed, their impact on Argentina and how plant genetic resources conservation mechanisms have evolved in the country, with the purpose of analyzing how a normative and institutional frame over plant genetic resources was shaped in Argentina.

Keywords: biodiversity; environmental legislation; environmental sovereignty; FAO; United Nations.

\section{Introducción}

En las últimas décadas ha comenzado a otorgarse gran importancia al rol que la diversidad de recursos genéticos podría tener en el desarrollo de la calidad y productividad de los cultivos que son fundamentales para la alimentación humana. Como consecuencia del desarrollo de la agricultura, la diversidad genética de las especies cultivadas sufrió una disminución, y esa pérdida de biodiversidad repercutió directa o indirectamente, en el bienestar del hombre (Pantuso, 2013). Esto se debe a que la selección realizada por agricultores durante miles de años de agricultura fue modificando las características genéticas de los cultivos al punto de que las plantas se han vuelto dependientes de la asistencia humana para su supervivencia. Además, el desarrollo intensivo de la agricultura en el último siglo condujo a una disminución mayor de la diversidad genética, toda vez que se concentró en pocos cultivos muy rentables.

Aunque el concepto de biodiversidad (o diver- sidad biológica) puede ser empleado con connotaciones diversas, se lo puede definir como la variabilidad de organismos vivos a cualquier nivel, ya sea dentro de cada especie, entre especies, o entre ecosistemas. Dentro de esa variabilidad, los recursos genéticos han cobrado también gran relevancia, pues la diversidad de información hereditaria disponible resulta fundamental para la generación de nuevas variedades de cultivos, por lo que su incidencia en la agricultura y la alimentación es clave.

La variabilidad genética puede resultar beneficiosa para proveer características (resistencias a cambios climáticos o virus, por ejemplo) que mejoren los cultivos disponibles, disminuyendo riesgos e incertidumbres productivas (Vilá \& Yacobaccio, 2013). Por ello, se han implementado diversos modos para conservar la diversidad de recursos genéticos que podrían tener utilidad para la agricultura y la alimentación: modos de conservación in situ (en el lugar donde se produce); y modos de conservación ex situ, entre los que se destacan los bancos de germoplasma, que contienen fundamentalmente 
semillas (León-Lobos et al., 2010; Pantuso, 2013).

La conservación y el uso de la biodiversidad se han convertido así en un tema presente en la agenda y en la normativa internacional en los últimos años. Dentro de este proceso global de valorización de la biodiversidad, en este trabajo nos concentraremos en una parte, la que hace a los recursos genéticos, más específicamente a los recursos fitogenéticos (los recursos genéticos de las plantas) de impacto en la agricultura y la alimentación.

En este trabajo ahondaremos en particular en el escenario que se presenta en la Argentina, país donde la agricultura resulta un sector fundamental de la economía. La pregunta que atraviesa al artículo es: ¿cómo se construyó un marco normativo e institucional que regula el acceso a los recursos fitogenéticos en la Argentina? El objetivo es analizar el desarrollo de una política de conservación y uso de recursos fitogenéticos, con relación a su marco institucional y a la incidencia de las normas nacionales e internacionales en la materia.

El abordaje del presente trabajo consiste en un estudio cualitativo, que combina entrevistas en profundidad a referentes de bancos de semillas, con documentos y otras fuentes primarias y secundarias relacionadas a recursos fitogenéticos. El artículo se estructura así en las siguientes secciones: en primer lugar, analizaremos cómo se desarrollaron las principales normas y acuerdos internacionales sobre el tema; luego veremos cómo impactaron en la Argentina y qué normas propias se han implementado al respecto; a continuación, analizaremos cómo se ha desarrollado la conservación de recursos fitogenéticos en la Argentina.

\section{Iniciativas globales sobre uso y acceso a recursos genéticos}

A medida que la industria de semillas comenzó a ganar importancia en los inicios del siglo XX, surgieron diversas iniciativas nacionales destinadas a regular el acceso y utilización de los recursos para la agricultura, como el acta de patentamiento que promulgó Estados Unidos en 1930. Las medidas multilaterales, no obstante, comenzaron a desplegarse algunas décadas más tarde.

Una de las primeras iniciativas globales surgió a partir del trabajo de la Asociación Internacional de Seleccionadores (ASSINSEL por sus siglas en Francés). Los miembros de la ASSINSEL celebraron en 1956 una conferencia abocada a desarrollar un "instrumento internacional novedoso para la protección de las obtenciones vegetales". Sobre la base de dicha conferencia se configuró luego la Unión para la Protección de las Obtenciones Vegetales (UPOV). Con la firma de 12 países y con organismos observadores como las Oficinas Internacionales Reunidas para la Protección de la Propiedad Intelectual (actual OMPI) y la Organización de las Naciones Unidas para la Alimentación y la Agricultura (FAO), se celebró en 1961 el Convenio Internacional para la Protección de las Obtenciones Vegetales. El convenio fue ratificado en 1968 y posteriormente varios países fueron sumados como miembros (Dutfield, 2011). Actualmente, la UPOV persigue tres objetivos: establecer las bases jurídico-administrativas y técnicas para la cooperación internacional, promover la aplicación de legislación local en los países miembro y hacer tareas de difusión al público en general (UPOV, 2017).

En forma paralela, las normas que regulan la preservación y el acceso a los recursos fitogenéticos 
fueron implementadas en las últimas décadas por los organismos internacionales nucleados en la Organización de las Naciones Unidas (ONU). El Consorcio de Centros Internacionales de Investigación Agraria (CGIAR), un grupo creado en 1971 por 18 países y organizaciones cuyo objetivo común era la lucha contra la hambruna, estableció en Roma en 1974 el Consejo Internacional de Recursos Fitogenéticos (IBPGR), un centro cuya función básica era la "conservación y uso de los recursos genéticos vegetales para el beneficio de las presentes y futuras generaciones" (Hanson et al., 1984; Gupta, 2008). Para ello, el IBPGR comenzó a coordinar bancos de semillas ya existentes, a construir nuevos, y a fomentar misiones para la recolección de germoplasma. Si bien este grupo no fue el primero del CGIAR dedicado a la conservación vegetal, el mismo nucleó y catalizó diversas iniciativas de recolección de semillas a nivel mundial. Los centros del CGIAR perfeccionaron las técnicas ex situ y marcaron un modelo a seguir de banco genético vegetal (CTA, 1992).

Casi una década después, en el año 1983, surge un importante instrumento legal internacional: la Organización de las Naciones Unidas para la Alimentación y la Agricultura (FAO) adopta el Compromiso Internacional sobre los Recursos Fitogenéticos (CIRF). El CIRF reivindicó la necesidad de conservar la variabilidad genética a fin de lograr mejoras en los cultivos a la vez que consideraba a los recursos fitogenéticos como un "patrimonio de la humanidad que hay que conservar y que debe estar libremente disponible para su utilización" (FAO, 1983). El documento de 1983 sentó las bases para la actual Comisión de Recursos Genéticos para la Alimentación y la Agricultura de la FAO, un espacio de discusión con representantes de varios países respecto a políticas estratégicas en biodiversidad para la alimentación y la agricultura.

Finalmente, la década de 1990 traería un nuevo marco legal a estas iniciativas. En 1991, la FAO reconoció los plenos derechos soberanos de los países sobre sus recursos fitogenéticos (FAO, 1991). La resolución reconocía el concepto de "herencia de la humanidad", tal como se aplica en el CIRF, pero lo deja "sujeto a la soberanía absoluta de los Estados sobre sus recursos fitogenéticos" (ibidem). $\mathrm{Al}$ año siguiente la Organización de las Naciones Unidas (ONU) ratificó esta última y otras iniciativas, a través del Convenio sobre la Diversidad Biológica. Legalmente, el convenio constituye un acuerdo marco, ya que traza metas y políticas generales sin marcar objetivos y líneas de acción precisas, dejando a cada parte libre de implementar las políticas a su voluntad (Glowka et al., 1996). Según sus principios, "los Estados tienen el derecho soberano de explotar sus propios recursos en aplicación de su propia política ambiental y la obligación de asegurar que las actividades que se lleven a cabo dentro de su jurisdicción o bajo su control no perjudiquen al medio de otros estados o de zonas situadas fuera de toda jurisdicción nacional" (ONU, 1992, art. 3).

De tal forma, el Convenio sobre la Diversidad Biológica reconoce la soberanía de los países para la explotación de sus recursos naturales, pero siempre bajo la responsabilidad legal de conservar su diversidad y utilizarla en forma sustentable, ya que si bien el derecho de explotación es soberano, "la conservación de la diversidad biológica es de interés común". De esta manera, permite e insta a los países a dictar sus propias leyes de regulación de la explotación de sus recursos conforme a los principios de protección ambiental internacionales. 
Además, artículos específicos del CDB plantean responsabilidad y obligaciones particulares a fin de llevar a cabo la conservación y la utilización sustentable (ONU, 1992, arts. 6, 8 y 10). Asimismo, se asume el compromiso del intercambio de información y la cooperación científico-tecnológica entre los firmantes. Esta sería la base legal para la construcción de políticas públicas y leyes especializadas en conservación fitogenética y al establecimiento de los modernos bancos de semillas nacionales como medio de conservación, así como a la regulación de sus prácticas.

De este modo, el Convenio sobre la Diversidad Biológica de la ONU de 1992 abre el espacio para la apropiación nacional de los recursos genéticos, desplazando la noción de patrimonio de la humanidad hacia un patrimonio de las naciones. No obstante, existían al momento dos antecedentes en las resoluciones de la asamblea general de Naciones Unidas de 1958 y 1962 donde se reconocía "el derecho de los pueblos y las naciones a la soberanía permanente sobre sus riquezas y recursos naturales" (ONU, 1958; 1962).

Al mismo tiempo, en este convenio se plasma la atribución de otro sentido a la conservación de la diversidad biológica: como un fin en sí mismo que debe ser preservado de las actividades humanas. Esto conduciría al posterior surgimiento de bancos de semillas ya no con fines únicamente productivos sino también preservacionistas. De este modo, según una clasificación realizada en función de sus objetivos y funcionamiento, es posible distinguir tipos de bancos de semillas: asistencialistas, productivistas y preservacionistas (Pellegrini \& Balatti, 2016). Las cuestiones vinculadas a la transferencia de material genético fueron las más ríspidas en el marco de las reuniones previas a la firma del convenio, y se manifiestan en el "tercer objetivo" del mismo, que aboga por la "participación justa y equitativa de los beneficios que se deriven de los

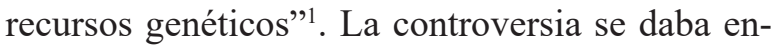
tre los países englobados en el "eje norte" (países altamente industrializados con menor biodiversidad) y los englobados en el "eje sur" (economías primarizadas con alta diversidad biológica dentro de sus fronteras) (Beyerlin, 2006). De hecho, la mera inclusión del "tercer objetivo" fue una hazaña para el "eje sur". Sin embargo, la propia laxitud del CDB llevó a que la inclusión de dicho objetivo no fuera más que una declaración, sin establecer mecanismos útiles para facilitar el acceso en forma justa y equitativa a los recursos genéticos (Greiber et al., 2012). Ello llevó en el año 2010 a la adopción del Protocolo de Nagoya, que reafirma y refuerza la posición de los países del eje sur, define en forma clara de qué se habla con "utilización" de recursos genéticos, establece nuevas obligaciones para los miembros del CDB y enfatiza la necesidad de proteger los conocimientos tradicionales (COP, 2010).

Atado a ello, el CDB también promovió la implementación de una herramienta legal que transparente la transferencia de material genético a través de los Acuerdos de Transferencia de Material (SMTA por sus siglas en inglés). Si bien las transferencias de material constituyen contratos privados entre el proveedor del material genético y quien lo recibe, los SMTA proveen de un marco legal con términos y condiciones prefijados y prenegociados en el contexto de la CDB (Tvedt, 2015). Otras iniciativas, como el Plan de Acción Mundial para

\footnotetext{
${ }^{1}$ Los objetivos del CDB, enumerados en su artículo primero, son tres: la conservación de la diversidad biológica, el uso sustentable de sus
} componentes y la participación justa y equitativa de los beneficios que se deriven de la utilización de los recursos genéticos. 
la conservación y la utilización sostenible de los recursos fitogenéticos para la alimentación y la agricultura (FAO, 1996) y el Segundo Plan de acción mundial para los recursos fitogenéticos para la alimentación y la agricultura (FAO, 2011) se celebraron con el fin de facilitar la aplicación del $\mathrm{CDB}$, unificando la línea planteada por la ONU. Entremedio, dos herramientas surgirían en el marco del Plan de Acción: el Tratado Internacional sobre los Recursos Fitogenéticos para la Alimentación y la Agricultura, y el Fondo Mundial para la Diversidad de Cultivos, ambas puestas en vigor durante el 2004. El primero acordó facilitar el intercambio de recursos genéticos entre países, en tanto que el segundo facilita mecanismos de financiamiento para los centros de conservación. Estas acciones buscaron lograr la aplicación práctica de los objetivos planteados en el CDB. Las iniciativas anteriormente mencionadas se esquematizan a modo de resumen en la Tabla 1.

TABLA 1 - Principales normas internacionales sobre recursos fitogenéticos.

\begin{tabular}{|c|c|c|c|}
\hline Instrumento & Año & Ente & Observaciones \\
\hline $\begin{array}{l}\text { Convenio Internacional para la Protec- } \\
\text { ción de las Obtenciones Vegetales }\end{array}$ & 1961 & UPOV & $\begin{array}{l}\text { Establecimiento de bases para proteger la propiedad intelectual de las } \\
\text { obtenciones vegetales }\end{array}$ \\
\hline Resolución 4 & 1989 & FAO & Reconocimiento de los "derechos del obtentor" descriptos por la UPOV \\
\hline Resolución 3 & 1991 & FAO & $\begin{array}{l}\text { Reconoce los plenos derechos soberanos de los países sobre sus propios } \\
\text { recursos fitogenéticos }\end{array}$ \\
\hline $\begin{array}{l}\text { Plan de Acción Mundial para la con- } \\
\text { servación y la utilización sostenible } \\
\text { de los recursos fitogenéticos para la } \\
\text { alimentación y la agricultura }\end{array}$ & 1996 & FAO & $\begin{array}{l}\text { Establecimiento de objetivos definidos, estrategia y un programa de acción } \\
\text { con el fin de cumplir lo establecido por la FAO en herramientas anteriores }\end{array}$ \\
\hline $\begin{array}{l}\text { Constitución del Fondo Mundial para } \\
\text { la Diversidad de Cultivos }\end{array}$ & 2004 & $\begin{array}{l}\mathrm{FAO} / \\
\text { CGIAR }\end{array}$ & $\begin{array}{l}\text { Constituir un fondo económico que garantice la aplicación del Plan de } \\
\text { Acción y demás iniciativas aquí citadas }\end{array}$ \\
\hline Protocolo de Nagoya & 2010 & $\begin{array}{l}\text { Conferencia } \\
\text { de las Partes } \\
\text { (COP) }\end{array}$ & Garantizar el "tercer objetivo" del CDB \\
\hline $\begin{array}{l}\text { Segundo Plan de Acción Mundial para } \\
\text { la conservación y la utilización sos- } \\
\text { tenible de los recursos fitogenéticos } \\
\text { para la alimentación y la agricultura }\end{array}$ & 2011 & FAO & Actualización del Plan Mundial 1996 \\
\hline
\end{tabular}




\section{Génesis de un marco normativo local}

A continuación, profundizaremos en el análisis de las normas e instituciones que se desplegaron en la Argentina en materia de conservación de recursos fitogenéticos, íntimamente ligados al contexto internacional y a los acuerdos globales anteriormente citados. Un resumen de las resoluciones y normas adoptadas en el país se sintetiza en la Tabla 2.

\subsection{Leyes sobre semillas en Argentina}

Durante el llamado "período agroexportador", que se extiende desde mediados del siglo XIX hasta 1930, la República Argentina vivió una etapa de sostenido crecimiento basado fundamentalmente en el comercio exterior agropecuario, ligado al aprovechamiento de los recursos naturales en función de la demanda global (Sábato, 1993). Sin embargo,

TABLA 2 - Principales normas argentinas vinculadas a los recursos fitogenéticos.

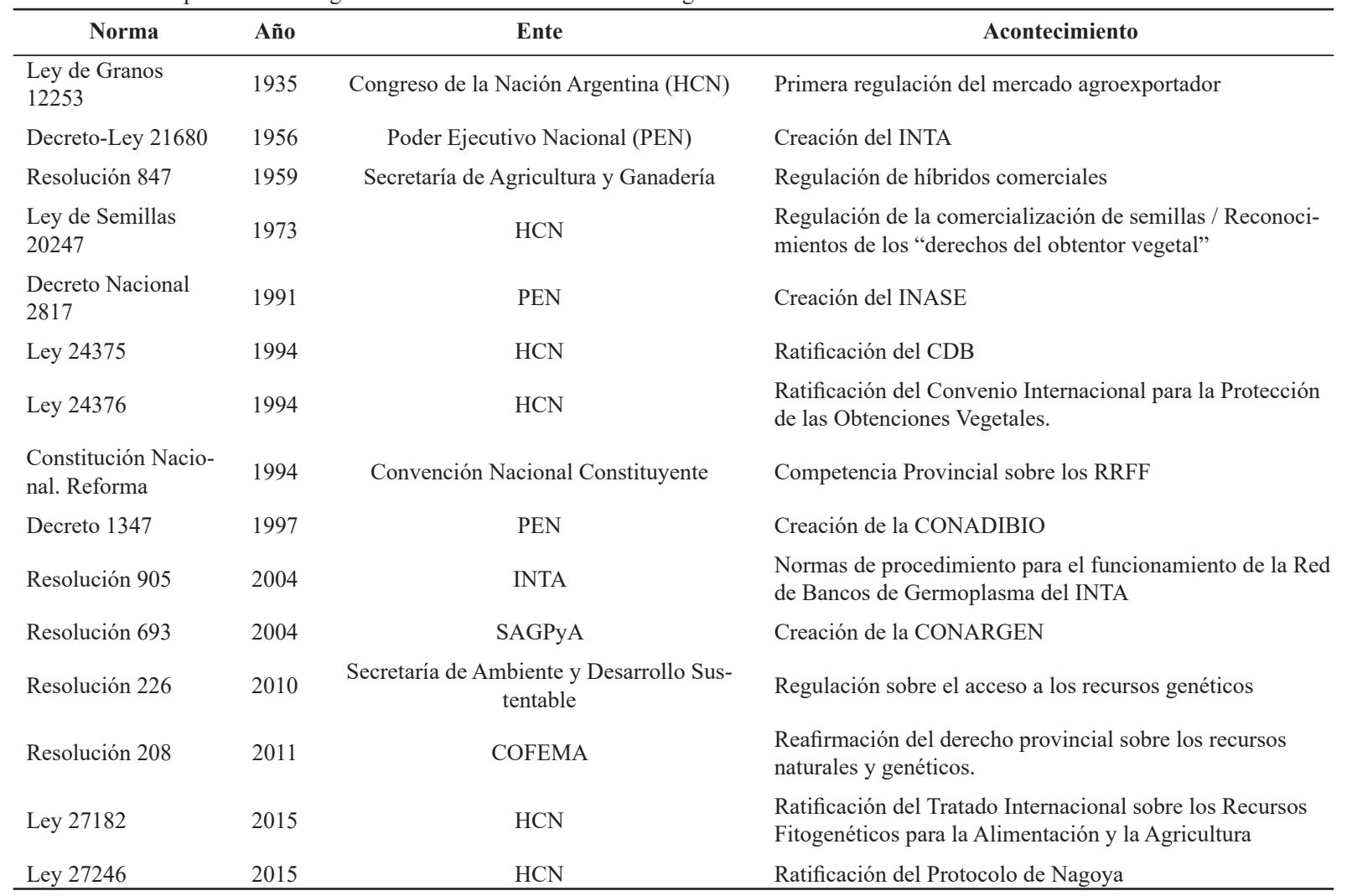


y luego del "crac" de la bolsa Neoyorkina en 1929, la denominada "gran depresión" estadounidense devenida en crisis mundial arrastraría a la baja a la totalidad de los valores financieros. Estos valores incluían commodities agropecuarias, de forma tal que los países agroexportadores debieron realizar grandes cambios en sus políticas públicas a fin de aumentar el intervencionismo estatal sobre el desregulado mercado agropecuario (Leavy \& Sáez, 2008; IEE, 2014). En tal sentido, se dicta en 1935 la Ley 12.253, conocida como "Ley de Granos", que en su capítulo denominado "Fomento de la Genética", buscaba estimular el aumento de la calidad y productividad de las variedades de cultivos. Para ello, regulaba el lanzamiento al mercado nuevas variedades de cultivos, evaluando la adaptabilidad de la semilla a diferentes climas y registrando a las instituciones participantes. En definitiva, el propósito era el de estimular un aumento de la productividad mediante la organización eficiente del sistema productivo (Hendel, 2010).

En el año 1959 la Secretaría de Agricultura y Ganadería, a través de la resolución 847 regula la inscripción de híbridos comerciales, creando dos categorías: de "pedigrí abierto" y "de pedigrí cerrado". La primera categoría enmarcaba a los híbridos públicos, que serían de libre disponibilidad, y la segunda a aquellos registrados por el sector privado, que serían guardados en secreto y por lo tanto de disponibilidad restringida, protegidos en forma similar a una patente. Esto facilitó la apropiación privada de recursos genéticos de origen público, ya que impedía explicitar el peso de las líneas públicas dentro de las creaciones privadas (Katz \& Bercovich, 1988; Gárgano, 2013).

En 1994, a través de la ley 24.376, Argentina adhirió al Convenio de 1978 de la UPOV (Gianni,
2010). Pero la protección legal sobre los desarrollos privados ya se había cristalizado en Argentina en 1973 con la Ley de Semillas y Creaciones Fitogenéticas, por la cual se reconocen los Derechos de Obtentores Vegetales. Según su artículo primero, la Ley "tiene por objeto promover una eficiente actividad de producción y comercialización de semillas, asegurar a los productores agrarios la identidad y calidad de la simiente que adquieren y proteger la propiedad de las creaciones fitogenéticas". Con la sanción de esta ley -aún vigente, aunque hay iniciativas para su reemplazo-, la Argentina se convirtió en el primer país de la región en disponer de una norma de este tipo, probablemente debido al desarrollo que tenía en el país la industria de semillas.

\subsection{El surgimiento del INTA}

A pesar de la temprana existencia de la Ley de Granos, las iniciativas estatales dirigidas a promover el mejoramiento de las semillas recién comenzaron a consolidarse a partir de los años '50. Precisamente en el año 1956 se sancionó el Decreto-Ley 21.680, que creó el Instituto Nacional de Tecnología Agropecuaria (INTA) con el fin de "impulsar, vigorizar y coordinar el desarrollo de la investigación y extensión agropecuaria y acelerar con los beneficios de estas funciones fundamentales la tecnificación y el mejoramiento de la empresa agraria y de la vida rural". El INTA comenzó sus actividades en 1958, en instalaciones que anteriormente pertenecían al Ministerio de Agricultura (INTA, 2006). Así, el INTA fue concentrando bajo su órbita los institutos agropecuarios que habían sido creados previamente, tales como la Estación Experimental de Pergamino (1912), el Instituto de 
Suelos (1943), el Instituto de Microbiología (1944) y el Instituto de Fitotecnia (1945), los cuales pasarían a depender del INTA (Bisang, 1994; Katz \& Bercovich, 1988) y a los cuales se sumarían nuevos centros de investigación y desarrollo en la materia.

A lo largo de su historia, el INTA fue atravesando diversos cambios, algunos debido al influjo de nuevas corrientes científicas, como los provocados por la irrupción de la biología molecular, que se tradujo en cambios en las disciplinas de origen de los investigadores del INTA (Pellegrini, 2013); otros, debido a los drásticos cambios en los contextos político-económicos del país, siendo la etapa de mayor dificultad para el INTA la década de 1990, cuando se redujo enormemente su cantidad de personal y hasta hubo un intento de privatización de la institución (Pellegrini, 2014). No obstante, el INTA continuó siendo la institución más importante de la Argentina en lo que se refiere a investigación y desarrollo para el sector agropecuario, considerando su extensión territorial y la gran diversidad de líneas de trabajo y grupos que lo componen, y se trata también de la institución que cobija a la red de bancos de germoplasma más importante del país.

\subsection{Impacto en la Argentina del CDB y normalización de los bancos de semillas}

En el año 1994 se ratificó localmente el Convenio sobre la Diversidad Biológica (CDB) mediante la Ley 24.375, creándose diversos organismos públicos nacionales para facilitar la implementación del convenio. Sin embargo, muchos de estos organismos comparten competencias u objetivos, e incluso componen la mesa directiva en varias de las iniciativas además de la propia. Tal complejidad ha redundado en idas y vueltas administrativas y legales a lo largo del tiempo.

A fin de garantizar la aplicación del CDB en el año 1997 se creó la CONADIBIO. Dicho organismo tiene la función de asesorar a la Secretaría de Ambiente y Desarrollo Sustentable (SAyDS) en la implementación del Convenio de Diversidad Biológica. La CONADIBIO, según se establece en su creación, "estará compuesta por especialistas técnicos y cuyas funciones serán de asesoramiento sobre la aplicación de políticas de estado en materia de biodiversidad siempre bajo los preceptos del Convenio sobre Diversidad Biológica" (PEN, 1997). Hoy día, la CONADIBIO está compuesta por diversos actores además de la Secretaría, entre ellos el Ministerio de Agricultura, el de Ciencia, los mencionados INTA, INASE y COFEMA, el CONICET, representantes de pueblos originarios, empresas, ONGs vinculadas al CDB y organizaciones profesionales y gremiales, entre otras.

Por otra parte, la Secretaría de Agricultura (SAGPyA) creó en 2004 y bajo su ámbito la Comisión Nacional Asesora en Recursos Genéticos para la Alimentación y la Agricultura (CONARGEN) con el fin de "asesorar a las autoridades en los temas inherentes a los recursos genéticos para la alimentación y la agricultura, tanto en el ámbito nacional como internacional" (SAGPyA, 2004). Esta comisión incluye tanto a miembros del INTA como del INASE y otros organismos, formando grupos ad hoc para abordar casos específicos (FAO, 2008). Luego, en 2010, el entonces Ministerio de Agricultura, Ganadería y Pesca aprobó su nueva estructura administrativa y creó en tal marco la Coordinación de Gestión Ambiental, pasando el CONARGEN a su órbita. En dicha Resolución se plantea explícitamente la "protección, conservación y utilización de 
la diversidad biológica y de los recursos genéticos para la alimentación y la agricultura" como línea de acción del organismo (MAGyP, 2010, Anexo II).

Por su parte, el INTA puso en marcha durante 2004 las normas de procedimiento para el funcionamiento de la Red de Bancos de Germoplasma del INTA, que reconocen a los bancos públicos de semillas como "un insumo básico para programas de mejoramiento tanto públicos como privados" y a los recursos genéticos como "estratégicos para el país" (INTA, 2004). Según el documento "es necesario que el Estado garantice la conservación y acceso a los mismos". Define también dos tipos de bancos de semillas: el banco "Activo" y el "Base", y brinda las recomendaciones básicas de manejo de los recursos, así como la normativa contractual para sesión de material a terceros. Para esto último, la herramienta legal necesaria es el universal Acuerdo de Transferencia de Material, cuyos términos particulares se encuentran detallados en las normas del INTA del 2004.

Por otra parte, la Secretaría de Ambiente emitió en 2010 la resolución 226, que regula y reglamenta el acceso a los recursos genéticos, según los objetivos establecidos por el CDB, definidos como "la conservación de la diversidad biológica, la utilización sostenible de sus componentes y la participación justa y equitativa en los beneficios que se deriven de la utilización de los recursos genéticos" (SAyDS, 2010). La norma cumple estrictamente lo determinado por el CDB. Para implementarlo determina que quien acceda al material genético alcanzado por la CDB, cualquiera sea su origen y con fines científicos o comerciales, debe solicitar el acceso formalmente a la Secretaría de Ambiente. La resolución también crea el "Registro de Acceso a los Recursos Genéticos", donde serán consignadas todas las solicitudes de material alcanzadas por la norma (SAyDS, 2010).

En 2015 el Congreso de la Nación Argentina refrendó localmente dos importantes acuerdos internacionales: el Tratado Internacional sobre los Recursos Fitogenéticos para la Alimentación y la Agricultura $^{2}$ y el Protocolo de Nagoya ${ }^{3}$.

\subsection{Reforma de la Constitución Nacional y controversia Nación-Provincias}

La reforma de la Carta Magna en 1994 también incluyó cuestiones vinculadas a la protección del medio ambiente, dándole jerarquía constitucional a la problemática. En particular, aborda la cuestión de la biodiversidad, a la cual establece como de competencia federal con complementación provincial:

Las autoridades proveerán a la protección de este derecho, a la utilización racional de los recursos naturales, a la preservación del patrimonio natural y cultural y de la diversidad biológica, y a la información y educación ambientales. (Art. $41 \mathrm{CN}$, párrafo 2)

Corresponde a la Nación dictar las normas que contengan los presupuestos mínimos de protección, $\mathrm{y}$ a las provincias, las necesarias para complementarlas, sin que aquellas alteren las jurisdicciones locales (Art. $41 \mathrm{CN}$, párrafo 3)

\footnotetext{
${ }^{2}$ Ley 27.182, sancionada el 23/09/2015, y promulgada el 05/10/2015. Disponible en: http://www.infoleg.gob.ar/infolegInternet/anexos/250000-254999/252910/norma.htm

${ }^{3}$ Ley 27.246, sancionada el 26/11/2015 y promulgada el 23/12/2015. Disponible en: http://servicios.infoleg.gob.ar/infolegInternet/anexos/255000-259999/257274/norma.htm
} 
Sin embargo, en el mismo texto, en el artículo 124 , se atribuye a las provincias el "dominio originario" sobre "los recursos naturales existentes en su territorio". Esto plantea una controversia en cuanto a los derechos y responsabilidades que las Provincias y la Nación tienen sobre los recursos fitogenéticos, y con ella cuánta injerencia tendrán los gobiernos provinciales en el almacenamiento de semillas propias de su territorio, ya que las competencias Nación-Provincia no se encuentran definidas en forma clara en los artículos precedentes. Este debate fue zanjado luego por la Corte Suprema de Justicia de la Nación (CSJN) en su interpretación sobre los controversiales artículos en el fallo "Magdalena Roca contra Provincia de Buenos Aires", donde reconoce plenamente la jurisdicción provincial en la materia:

Corresponde reconocer a las autoridades locales la facultad de aplicar los criterios de protección ambiental que consideren conducentes para el bienestar de la comunidad para la que gobiernan, así como valorar y juzgar si los actos que llevan a cabo sus autoridades, en ejercicio de poderes propios, afectan el bienestar perseguido, porque si bien la Constitución Nacional establece que le cabe a la Nación 'dictar las normas que contengan los presupuestos mínimos de protección', reconoce expresamente las jurisdicciones locales en la materia, las que no pueden ser alteradas (CSJN, 2012)

Para Sabsay, este nuevo esquema sumado a otras normativas ha generado una superposición de normas y, en especial, de competencias entre organismos nacionales y locales, configurando una especie de federalismo "de imposición" (Sabsay \& Di Paola, 2002).El Consejo Federal de Medio Ambiente (COFEMA), conformado en 1990 y autodefinido como "el espacio natural de encuentro federal para abordar los problemas y las soluciones del medio ambiente de la República Argentina" reafirmó en el año 2011 los artículos 41 y 124 de la Carta Magna (COFEMA, 2011).

\subsection{La creación del INASE}

Si bien la Ley de Semillas data de 1973, su autoridad de aplicación surgiría 18 años después, con la creación del Instituto Nacional de Semillas (INASE), un órgano descentralizado bajo la órbita de la Secretaría de Agricultura, Ganadería, Pesca y Alimentación (SAGPyA) 4 .

Como autoridad de aplicación de la Ley de Semillas, el INASE debe ejercer el poder de policía para el cumplimiento de ésta, certificando la calidad de las semillas destinadas a siembra, plantación o propagación, cualquiera sea su origen, y registrando la propiedad intelectual de las semillas (tanto las obtenidas por cruzamiento tradicional como por biotecnología) así como asesorar en normativa adicional vinculada a la Ley.

Respecto a las especies salvajes, no mencionadas en forma explícita en la Ley, una de las medidas más importantes del INASE fue la creación, en 2006, del Listado Nacional de Especies Vegetales Nativas. En dicho listado pueden incluirse "todos aquellos recursos fitogenéticos nativos que cuentan

\footnotetext{
${ }^{4}$ No sólo la creación del INASE fue tardía (en relación a la Ley de Semillas), sino que el instituto fue disuelto como órgano descentralizado en el año 2000 mediante el Decreto 1104, pasando sus competencias directamente a la SAGPyA. En 2003, dicho Decreto fue derogado, ratificando la vigencia de lo establecido en la Ley de Semillas y volviendo a la concepción del INASE como órgano descentralizado (Ley 25.845). Actualmente se encuentra en funciones.
} 
con valor para la alimentación y la agricultura, la forestación, aptitud ornamental y aplicación industrial, en sus diferentes sectores". De esta forma permite proteger también los cultivares tradicionales de interés productivo y origen nativo de la "apropiación ilegítima" por su utilidad "presente o futura". También consideraba necesario reconocer "la tarea ancestral de quienes han venido manteniendo y mejorando empíricamente estos recursos en forma comunitaria", ya que existen muchas variedades que fueron desarrolladas por comunidades campesinas y que se encuentran fuera del circuito comercial.

\section{Conservación de recursos fitogenéticos en la Argentina}

Así como anteriormente dimos cuenta de los aspectos normativos vinculados a los recursos genéticos, es también oportuno mostrar la dinámica que ha presentado en los hechos la conservación de recursos fitogenéticos en Argentina.

El desarrollo de la agricultura moderna en la Argentina se produjo fundamentalmente a partir de las semillas que trajeron los agricultores inmigrantes desde mediados del siglo XIX. La falta de técnicos e infraestructura estatal dio lugar a que fuera el propio impulso de los agricultores lo que expandiera el uso de los cultivos. Para mejorar el rendimiento de los cultivos, y en especial del trigo, en 1912 el Ministerio de Agricultura contrató al fitomejorador inglés Guillermo Backhouse, quien realizó selecciones genealógicas y cruzamientos, elevando el rendimiento del trigo (Nisi, 1983). Con esta contratación se inició una etapa de mejoramiento de los cultivos a partir de la llegada al país de varios fitomejoradores formados en el exterior (entre los que no sólo se destaca Backhouse, sino también el alemán Enrique Klein, quien se instalaría en el país en 1919), los que a su vez formarían discípulos locales, abriendo el camino para el fitomejoramiento en la Argentina. Estos mejoradores, una vez radicados en el país, abrieron sus propios criaderos de semillas, de modo que para 1930 comenzaban a desplegarse ciertas capacidades en conservación y mejoramiento genético de semillas en el ámbito privado (Gutiérrez \& Penna, 2004).

En definitiva, en la década de 1930 se forman las colecciones de germoplasma de trigo, maíz y maní, con el establecimiento de programas de mejoramiento de los principales cultivos por parte de los colonos europeos y los primeros fitomejoradores argentinos (Clausen et al., 1995). Posteriormente se conforman las colecciones de sorgo, girasol y algodón.

En 1935, a pesar de la sanción de la citada "Ley de Granos", las iniciativas estatales dirigidas a promover el mejoramiento de las semillas eran escasas y demoraron en consolidarse. Si bien, por ejemplo, los trabajos de mejoramiento por hibridación de maíz comenzaron en la década de 1920, recién con el advenimiento de los primeros maíces híbridos a partir de los años 50 el sector público consolidó su actividad en la materia, sobre todo gracias a las tareas de mejoramiento de la Estación Experimental de Pergamino (Rossi, 2007).

Hacia los años 1950, sin embargo, la evolución del panorama de variedades de cultivos en la Argentina era desalentador, lo que llevó a que durante la década siguiente se implementaran una serie de convenios con organismos internacionales para abastecer de germoplasma exótico y contribuir al mejoramiento de variedades locales, lo que permitió obtener variedades con mayores ventajas y rendi- 
miento (Giancola, 2003). Así es como se estableció un Programa Cooperativo Internacional entre el INTA y el Centro Internacional de Mejoramiento de Maíz y Trigo (CIMMYT) para desplegar planes de mejoramiento de maíz y trigo en las Estaciones experimentales de Pergamino y Marcos Juárez.

Las primeras colecciones de semillas, es decir, los primeros esfuerzos deliberados de conservación de semillas en el país, fueron obra fundamentalmente de los criaderos privados, quienes buscaban así ampliar la variedad de semillas para poder realizar cruzas y obtener nuevas variedades que rindieran mejor en sus respectivas zonas de cultivo. La escasa infraestructura desarrollada para la conservación de semillas hacía que la misma fuera limitada, en parte debido a que sin ningún tratamiento ni condiciones especiales, las semillas pueden conservarse sólo alrededor de 2 años y luego pierden viabilidad. Con la creación de un banco público de semillas habrían de cambiar esas condiciones.

\subsection{Banco público de semillas}

El INTA, a través de su red de bancos de semillas, guarda especies que sean de interés para la agricultura y la alimentación.

En 1969 la Estación Experimental de Pergamino, ubicada en la Provincia de Buenos Aires y perteneciente al Instituto Nacional de Tecnología Agropecuaria, creó el Banco de Germoplasma de Maíz, constituyéndose en el banco público de semillas más antiguo del país. Al poco tiempo, en 1970, se creó en la Estación Experimental de Balcarce el Banco de Germoplasma de Papa. Las actividades comenzaron con la habilitación de una cámara fría que permitía conservar por mucho tiempo las semillas.

Luego del banco de Pergamino, se creó el de papa en Balcarce, y luego se sucedieron otros: soja y trigo en Marcos Juárez (Córdoba); maní, sorgo y girasol en Manfredi (Córdoba); algodón en Chaco; poroto en Salta; cítricos en Concordia (Entre Ríos); yerba mate y té en Cerro Azul (Misiones), entre otros. La red de bancos del INTA se creó en 1988 gracias al aporte del gobierno de Italia, que donó cámaras de frío para guardar las semillas. Según el curador del banco de semillas de Pergamino, es probable que dicho aporte del gobierno italiano se haya realizado especulando con recibir un beneficio en algún momento, aunque lo cierto es que otorgaron esos equipos que aún funcionan hoy en día sin recibir nada a cambio, o al menos sin recibir germoplasma a cambio 5 .

Sin embargo, la participación de países desarrollados en los orígenes de los bancos de semillas en América Latina no es puramente casual ni altruista. Cuando comenzaron a implementarse los bancos de semillas en la Argentina, sus responsables debieron capacitarse en la conservación de recursos genéticos. Para ello, fueron fundamentales los cursos que otorgaba JICA (Agencia de Cooperación Internacional del Japón) en América Latina. Japón es un país que no tiene muchos recursos naturales, y no obstante tiene bancos de semillas que están entre los más completos que hay en el mundo. Cuando comenzó a promoverse a nivel global la implementación de bancos de semillas, hacia la década del ' 80 , no había marcos establecidos para el flujo de germoplasma entre países. En ese momento no había acuerdos de transferencia de material, no había

${ }^{5}$ Información obtenida en la entrevista a referente del Banco de Germoplasma de Pergamino. 
limitaciones a este tipo de acciones. Entonces, desde JICA daban cursos, ayudaban a instalar los bancos de germoplasma, hacían colectas, y a cambio se llevaban la mitad de lo que recolectaban. Los acuerdos consistían fundamentalmente en eso: ellos hacían colectas y se llevaban la mitad de lo que colectaban. Esto ocurría en toda América Latina ${ }^{6}$.

De este modo, los bancos de semillas del INTA asumieron la tarea de recolectar y conservar las semillas que se encontraban dentro de su competencia, procurando a su vez adquirir nuevos conocimientos en el tema. Así, con el objetivo de investigar modos de almacenamiento destinados a la conservación de recursos genéticos vegetales, el Banco de Germoplasma de Pergamino del INTA comenzó en 1983 a realizar ensayos con semillas en sitios naturales muy distintos: en la Antártida Argentina y en la región de la Puna en la provincia de Jujuy, al norte de Argentina (Rosso \& Ferrer, 2007). De este modo, 25 años antes de que en Noruega se construyera la Bóveda Global de Semillas de Svalbard, el INTA exploraba en la Antártida condiciones similares para conservar las semillas a muy largo plazo y sin costo de mantenimiento.

En 1988 se formalizó la Red de Bancos de Germoplasma del INTA, articulando a los bancos que existían en la institución. En 1991 se creó el Banco Base del INTA, en su sede de Castelar, en la Provincia de Buenos Aires. El resto de los bancos del INTA siguieron funcionando como bancos activos.

El INTA no conserva cualquier tipo de semillas, sino las que se encuentran vinculadas a la agricultura y la alimentación. A su vez, la distri bución geográfica de sus bancos de semillas no es azarosa: los bancos de semillas propios de determinado cultivo se establecen en la zona donde ese cultivo es predominante (Gittins, 2009). Esto pone en evidencia que la función de estos bancos es incidir en la producción agrícola: conservar semillas para usarlas en las labores de fitomejoramiento de la zona. De este modo, tanto la primera etapa de bancos privados como la segunda etapa caracterizada por los bancos públicos de semillas, le imponen a los bancos de semillas una utilidad dirigida a contribuir al mejoramiento de cultivos para ser empleados en la agricultura, es decir, presentan un perfil productivista.

A comienzos de la década de 1990 se abrió una nueva etapa, bajo el influjo de nuevas normas y resoluciones de organismos internacionales. Como señalamos anteriormente, en estas resoluciones, encabezadas por la ONU, se reflejó un nuevo paradigma en torno a los recursos biológicos, ponderando su soberanía nacional y la necesidad de su preservación de las actividades del hombre. En este marco, en 1993 comenzó a funcionar el Banco Base de semillas del INTA. Este banco se diferencia de los otros bancos del INTA, pues su función ya no es la de vincularse activamente con los fitomejoradores para contribuir en la producción de nuevas variedades de cultivos, sino que el Banco Base tiene la misión de conservar semillas a largo plazo, buscando mantener la integridad genética del germoplasma a través del tiempo ${ }^{7}$.

De este modo, la red de bancos de semillas del INTA - que conserva aproximadamente el 95\% del

\footnotetext{
${ }^{6}$ Información obtenida en la entrevista a referente del Banco de Germoplasma de Pergamino.

${ }^{7}$ Información obtenida en la entrevista a curadora del Banco Base de Germoplasma del INTA. El Banco Base de germoplasma, que está en el Instituto de Recursos Biológicos del INTA Castelar, lo que hace es conservar semillas a largo plazo. Se trata de semillas ortodoxas, es decir, semillas que se pueden secar y ser conservadas a $-20^{\circ} \mathrm{C}$ a largo plazo (Clausen et al., 2008).
} 
germoplasma disponible en instituciones oficiales en el país- distingue entre sus Bancos Activos y su Banco Base. En total, son 9 bancos activos, además de otras 12 colecciones distribuidas en diversas áreas ecológicas, y un Banco Base que mantiene un duplicado de todo (INTA, 2012).

Los bancos activos se dedican fundamentalmente a conservar semillas y utilizarlas para contribuir al mejoramiento de los cultivos empleados en la agricultura; mientras que el Banco Base se concentra en la conservación de semillas a largo plazo, sin vinculación con otros usuarios. El sistema de bancos de semillas del INTA surgió emulando a la red de bancos de otros países, en particular a la de Estados Unidos ${ }^{8}$. El principio de los bancos de germoplasma es que siempre todo material tiene que estar duplicado, tiene que estar en otro lugar también. De ese modo, si ocurre algún inconveniente en el banco activo, siempre hay un resguardo en otro lugar, donde se conservan las semillas a largo plazo.

Los bancos activos de semillas involucran un flujo de germoplasma con el entorno, a diferencia del Banco Base: intercambios con otros bancos de germoplasma, intercambios con investigadores, con mejoradores, o con comunidades. Un ejemplo que mencionan en el INTA de vinculación con una comunidad local se dio con el caso del maíz, en Catamarca, en la localidad de Belén, donde había unas comunidades indígenas que habían perdido variedades de maíz que habían tenido sus abuelos; no las mantuvieron en el tiempo y se perdieron. Entonces, el Banco de Pergamino, que sí tenía esas variedades, le entregó semillas a la comunidad ${ }^{9}$.Por otro lado, cabe señalar que la disputa por la apro- piación de las semillas está en pleno proceso de desarrollo. En efecto, a partir de la instauración de la posibilidad de que los estados nacionales puedan disponer del patrimonio sobre los recursos fitogenéticos, el INTA dispuso la conformación de convenios para el intercambio de semillas que poseen sus bancos, a través de acuerdos de transferencia de material. Estos convenios están en proceso de discusión y reelaboración, lo que no está exento de conflictos, pues, por ejemplo, investigadores del INTA que anteriormente accedían a las semillas de los bancos sin restricciones, suelen manifestar su disconformidad por las trabas que emergieron con la implementación de estos convenios. A su vez, como se expuso anteriormente, la apropiación de las semillas encuentra a nivel local una particularidad, y es que los recursos naturales, según la Constitución de 1994, pueden interpretarse como patrimonio de las provincias, lo cual genera disputas y dificultades, incluso a la hora de recolectar muestras para ser incluidas en los bancos de semillas. A esto se agregan las tensiones entre países, pues la disputa por la forma en que se producen los intercambios de semillas, la ratificación de normas internacionales, etc., suele enfrentar a la Secretaría de Ambiente de la Nación con sus pares de otros países.

Antes de la citada resolución 226/10 no había un modo reglamentado de realizar las colectas de semillas. Ahora se pide autorización a las provincias para realizar las colectas. Considerando que la jurisdicción del manejo de recursos naturales correspondería a las provincias a partir del año 1994, uno de los problemas que emerge es ¿qué hacer con las colecciones previas? Si no hubo consentimiento

\footnotetext{
${ }^{8}$ Entrevista a responsable del Banco Base de Germoplasma del INTA. ${ }^{9}$ Ibídem.
} 
firmado por las provincias de origen en su momento, para realizar intercambios con el exterior ¿deberían tener autorización de las provincias? Así, los bancos de semillas encuentran diversas tensiones actuales entre las normas que los regulan y sus prácticas.

\section{Conclusiones}

En un marco global de creciente importancia otorgada a la diversidad de recursos genéticos, en este trabajo nos propusimos indagar en el modo en que se fue construyendo un marco normativo e institucional para regular el acceso a los recursos fitogenéticos en la Argentina, a fin de analizar el modo en que van tomando forma las políticas relacionadas con su conservación y uso.

En tal sentido, es posible distinguir diversas etapas en el desarrollo de dicho marco. Una primera etapa de los bancos de semillas en la Argentina (a inicios del siglo $\mathrm{XX}$ ) está signada por su carácter privado, pues se trata de colecciones de semillas emprendidas por los mejoradores que buscaban generar nuevas variedades de cultivos realizando cruzas con variedades obsoletas que guardaban. Una segunda etapa se caracteriza por el ingreso del sector público en la labor de conservación de semillas (Clausen et al., 1995). La creación del Banco de Germoplasma de Maíz en la Estación Experimental de Pergamino, en el INTA, marca el inicio de esta etapa. El INTA, que inició sus funciones en 1958, se creó con la misión de contribuir al desarrollo agropecuario argentino, y fue asumiendo un rol central en lo que se refiere a bancos de semillas en el país.

Las normas nacionales e internacionales en la materia fueron fundamentales para promover y fijar el marco de funcionamiento de estas instituciones. No obstante, en la actualidad hay aspectos de su funcionamiento que ponen en tensión la coexistencia de diversas normas y puede llevar a su reformulación, toda vez que el carácter nacional del banco de semillas, junto con la potestad provincial que existe en la Argentina sobre las recursos biológicos, sumado a los frecuentes intercambios internacionales de materiales biológicos, genera múltiples tensiones.

Por otro lado, el desarrollo de los bancos de semillas no necesariamente indica que su uso acompaña su crecimiento. Así, en el 2005 se utilizaba solamente el $6,4 \%$ de la variabilidad genética conservada en la Red de Bancos de Germoplasma de INTA (Paredes et al., 2010) ${ }^{10}$.

Los modernos bancos de semillas constituyen en definitiva un tipo de institución reciente, que requiere de una mayor consideración por parte de las ciencias sociales. Diversas aristas jurídicas, sociales, políticas, económicas y biológicas, atraviesan el desarrollo y funcionamiento de los bancos de semillas. Al haber descrito aquí el modo en que se fue desplegando un marco normativo e institucional en torno a este objeto, esperamos haber contribuido al estudio de los bancos de semillas. Muchas de las tensiones y conflictos en torno al uso de los recursos fitogenéticos se encuentran delimitados por el marco normativo e institucional que los regula, cuyo mapa resulta insoslayable para su comprensión. Futuros trabajos deberán contemplar, también, el modo en que se despliegan las prácticas de los bancos de semillas, observando los actores que hacen uso de los mismos y sus modos concretos de funcionamiento.

\footnotetext{
${ }^{10}$ La utilización de los recursos genéticos contenidos en el banco de germoplasma varía según la especie de que se trata, pudiendo representar alrededor de un 2\% en papa, sorgo o maní, hasta un $40 \%$ en algunas especies forrajeras (Paredes et al., 2010).
} 


\section{Agradecimientos}

Esta investigación fue posible gracias al apoyo del CONICET. También fue financiada por la Agencia Nacional de Promoción Científica y Tecnológica a través del PICT-2011-1713. La misma fue realizada en Argentina, entre marzo de 2012 y octubre de 2015.

\section{Referencias}

Beyerlin, U. Bridging the north-south divide in international environmental law. Heidelberg Journal of International Law (ZaöRV), 66, 259-296, 2006.

Bisang, R. Industrialización e incorporación del progreso técnico en la Argentina. Documento de Trabajo 54. Buenos Aires: CEPAL, 1994.

CTA. IBPGR: International Board for Plant Genetic Resources, 1992. Technical Centre for Agricultural and Rural Cooperation (CTA): SPORE Bulletin $\mathrm{N}^{\circ} 38$. Recuperado el 9 de abril de 2015, de http://collections.infocollections.org/ ukedu/en/d/Jcta38e/9.1.html

Clausen, A. M.; Ferrer, M. E.; Gómez, S.; Tillería, J. Argentina: Informe nacional para la conferencia técnica internacional de la FAO sobre los recursos fitogenéticos (Leipzig, 1996), 1995. Buenos Aires. Recuperado el 10 de abril de 2015, de http://www.fao.org/fileadmin/templates/agphome/ documents/PGR/SoW1/americas/ARGENTIN.pdf

Clausen, A. M.; Ferrer, M. E.; Formica, M. B. Situación de los recursos fitogenéticos en la Argentina. II Informe nacional 1996-2006, 2008. Argentina: Ediciones INTA.

COFEMA. Reafirmación de derechos sobre los recursos naturales y genéticos. Consejo Federal de Medio Ambiente, 2011. Resolución 208. Sancionada el 16/03/2011.

COP. COP 10 Decision X/01. Convention on Biological Diversity. 2010. Recuperado el 3 de abril de 2017 de https:// www.cbd.int/decision/cop/default.shtml?id=12267

CSJN. Roca, Magdalena c/ Buenos Aires, Provincia de s/ inconstitucionalidad 16/05/1995 - Fallos: 318:992. Secretaría de Jurisprudencia de la Corte Suprema de Justicia de la Nación: Derecho Ambiental. Buenos Aires: Corte Suprema de Justicia de la Nación, 2012.

Dutfield, G. Alimentation, diversité biologique et propriété intellectuelle: le rôle de l'Union internationale pour la protection des obtentions végétales (UPOV). Bureau Quaker auprès des Nations Unies, 2011. Recuperado el 27 de marzo de 2017 de http://www.quno.org/sites/default/files/ resources/Alimentation, $\% 2 \mathrm{Bdiv} \% 2 \mathrm{Bbiol} \% 2 \mathrm{~B} \% 2 \mathrm{~B} \% 2 \mathrm{Bali}$ mentation\%2B-\%2BUPOV\%2BQUNO.pdf

FAO. Resolución 8/1983, 1983. Recuperado el 29 de septiembre de 2015, de http://www.fao.org/fileadmin/templates/nr/documents/CGRFA/Resolution8-83_s.pdf

FAO. Resolución 3/1991, 1991. Recuperado el 29 de septiembre de 2015, de http://www.fao.org/fileadmin/templates/nr/documents/CGRFA/Rightsofnations_s.pdf

FAO. Plan de Acción Mundial para la Conservación y la Utilización Sostenible de los Recursos Fitogenéticos para la Alimentación y la Agricultura. Cuarta Conferencia Técnica Internacional sobre los Recursos Fitogenéticos. Leipzig, Alemania, Junio 1996.

FAO. Informe sobre el Establecimiento del Mecanismo y el Estado de Aplicación del Plan de Acción Mundial en la Argentina, 2008. Argentina. Recuperado el 5 de mayo de 2015, de http://www.fao.org/docrep/013/i1500e/Argentina.pdf

FAO. Segundo Plan de acción mundial para los recursos fitogenéticos para la alimentación y la agricultura, 2011. Roma, Italia. Recuperado el 5 de mayo de 2015, de http:// www.fao.org/docrep/015/i2624s/i2624s00.pdf

Gárgano, C. Ciencia y dictadura: producción pública y apropiación privada de conocimiento científico-tecnológico. Dinámicas de cooptación y transferencia en el ámbito del Instituto Nacional de Tecnología Agropecuaria (INTA) durante la última dictadura cívico-militar argentina (19761983). Crítica y Emancipación, 5 (10), 131-170, 2013.

Giancola, S. I. Determinantes de la productividad y eficiencia de la investigación y desarrollo agrícola: el caso de la obtención de nuevos cultivares de trigo y soja. Tesis de Maestría en Economía Agraria, FAUBA, 2003. 
Gianni, C. El Convenio UPOV 1978 y la Legislación Argentina - cuadro comparativo. In: Gianni, C. (Coord.). Distintas facetas de las variedades vegetales. Buenos Aires: INASE, 70-81, 2010.

Gittins, C. Conservación de recursos genéticos. Revista Fruticultura \& Diversificación, 61, 10-15, 2009.

Glowka, L.; Burhenne-Guilmin, F.; Synge, H. Guía del Convenio sobre la Diversidad Biológica. Gland y Cambridge: UICN, 1996.

Greiber, T.; Peña Moreno, S.; Åhrén, M.; Nieto Carrasco, J.; Chege Kamau, E.; Cabrera Medaglia, J.; Oliva, M. J.; Perron-Welch, F.; Ali, N.; Williams, C. An Explanatory Guide to the Nagoya Protocol on Access and Benefit-sharing. IUCN Environmental Policy and Law Paper No. 83, Gland. 2012

Gupta, P. K. Elements of Biotechnology. New Delhi: Rastogi Publications, 2008.

Gutiérrez, M.; Penna, J. A. Derechos de obtentor y estrategias de marketing en la generación de variedades públicas y privadas. INTA, Documento de Trabajo 31, 2004. Recuperado el 19 de mayo de 2015, de http://inta.gob.ar/ documentos/derechos-de-obtentor-y-estrategias-de-marketing-en-la-generacion-de-variedades-publicas-y-privadas/ at_multi_download/file/dt_31.pdf

Hanson, J.; Williams, T.; Freund, R. Institutes conserving crop germplasm: The IBPGR global network of genebanks. Roma: IBPGR Executive Secretariat, 1984.

Hendel, V. Genealogía de la semilla. Regulación y escasez en la región pampeana de la argentina. Nómadas. Revista Crítica de Ciencias Sociales y Jurídicas, 27(3), 351-367, 2010.

IEE. Juntas Nacionales de Granos: lejos de los nuevos desafíos mundiales. Instituto de Estudios Económicos de la Bolsa de Cereales de Buenos Aires, 2014. Recuperado el 7 de mayo de 2015, de http://bolsadecereales.com.ar/greenstone/collect/bolcer/index/assoc/HASH0123.dir/Juntas $\% 20$ Nacionales\%20Granos.pdf

INTA. Red de Bancos de Germoplasma del INTA. Normas de Procedimientos. Resolución 905. INTA, 2004.

INTA. INTA, medio siglo al servicio del campo argentino. Buenos Aires: INTA, 2006.
INTA. Una red que protege la biodiversidad. INTA Informa, XI(127), 2012.

Katz, J.; Bercovich, N. Innovación genética, esfuerzos públicos de investigación y desarrollo y la frontera tecnológica internacional: nuevos híbridos en el INTA. Desarrollo Económico, 28(110), 209-243, 1988.

Leavy, S.; Sáez, F. F. Intervención del Estado en el comercio granario: Los casos de la Junta Nacional de Granos y de la Oficina Nacional de Control Comercial Agropecuario. Asociación Argentina de Economía Agraria. Agosto de 2008.

León-Lobos, P.; Seguel, I.; Condón, F. Conservación ex situ de los recursos fitogenéticos. In: Berretta, A. (Coord.). Estrategia en los recursos fitogenéticos para los países del cono sur. Montevideo: PROCISUR/IICA, 41-58, 2010.

MAGyP. Resolución No 395. Ministerio de Agricultura, Ganadería y Pesca. Buenos Aires, 5/10/2010.

Nisi, J.E. Producción de trigo en la Argentina. Simposio sobre fitomejoramiento y producción de cereales, del 7 al 12 de noviembre de 1983. Marcos Juárez: INTA, pp. 8-23. Recuperado el 20 de mayo de 2015, de http://pdf.usaid.gov/ pdf_docs/pnaar205.pdf

ONU. Resolución 1314 (XIII) de la Asamblea General. Firmado el 12/12/1958.

ONU. Resolución 1803 (XVII) de la Asamblea General. Firmado el 14/12/1962.

ONU. Convenio sobre la Diversidad Biológica. Firmado el 05/06/1992. Entrada en vigor 29/12/1993. Río de Janeiro: ONU.

Pantuso, F. S. Conservación de los recursos fitogenéticos. Buenos Aires: Ciccus, 2013.

Paredes, M.; Becerra, V.; Condón, F.; Germán, S.; Barbieri, R. L. Utilización de los recursos fitogenéticos. In: Berretta, A. (Coord.), Estrategia en los recursos fitogenéticos para los países del cono sur (pp. 97-116). Montevideo: PROCISUR/IICA, 2010.

Pellegrini, P. A. Del campo al laboratorio. La institucionalización de la biología molecular en Argentina. Scientiae Studia. Revista Latino-Americana de Filosofia e História da Ciência, 11(3), 531-556, 2013. 
Pellegrini, P. A. Argentina: evolución del presupuesto y del personal del INTA (1958-2010). Realidad Económica, 285, 99-122, 2014.

Pellegrini, P. A.; Balatti, G. Noah's arks in the XXI century. A typology of seed banks. Biodiversity and Conservation, 25(13), 2753-2769, 2016.

PEN. Decreto 1347 del Poder Ejecutivo Nacional, 1997. Boletín oficial, 02/03/2010. Recuperado el 5 de octubre de 2015, de http://conadibio.ambiente.gob.ar/?aplicacion=normativa $\&$ IdNorma $=426 \&$ IdSeccion $=331$

Rossi, D. Evolución de los cultivares de maíz utilizados en la Argentina. Revista Agromensajes, 22, 3-10, 2007.

Rosso, B. S.; Ferrer, M. E. Conservación de semillas en ambientes naturales. In: Clausen, A.; Condón, F.; Berretta, A. (Eds.). Avances de Investigación en Recursos Genéticos en el Cono Sur II. Montevideo: PROCISUR, 143-146, 2007.

Sabsay, D. A.; Di Paola, M. E. El Federalismo y la Nueva Ley General de Ambiente. Anales de Legislación Argentina, Boletín Informativo, 32, 47-54, 2002.

Sábato, H. Estructura productiva e ineficiencia del agro pampeano, 1850-1950: un siglo de historia en debate. In: Bonaudo, M.; Pucciarelli, A.R. (Comps.). La problemática agraria: Nuevas aproximaciones, III. Buenos Aires: Centro Editor de América Latina, 7-49, 1993.

SAGPyA. Resolución N 693. Buenos Aires: SAGPyA, 2004.

SAyDS. Resolución 226. Buenos Aires: Secretaría de Ambiente y Desarrollo Sustentable, 2010.

Tvedt, M. W. Changes in the Plant Treaty - How Can Benefit Sharing Happen and the Link to Intellectual Property Rights - Assessing the Mutually Supportiveness. Law Environment and Development Journal, 11(1), 3-16, 2015.

UPOV. ¿Qué hace la UPOV?, 2017. Recuperado el 27 de marzo de 2017 de http://www.upov.int/about/es/faq. html\#QG20.

Vilá, B.; Yacobaccio, H. Domesticación: moldeando la naturaleza. Buenos Aires: Eudeba, 2013.

\section{Entrevistas}

Responsable del Banco Base de Germoplasma del INTA, 3 de mayo de 2012.

Curadora del Banco Base de Germoplasma del INTA, 25 de agosto de 2011.

Curador del Banco de Germoplasma de Pergamino, 10 de octubre de 2013.

Referente del Banco de Germoplasma de Pergamino, 10 de octubre de 2013. 